Article

\title{
Orness For Idempotent Aggregation Functions
}

\author{
Leire Legarreta $^{1}$, Inmaculada Lizasoain ${ }^{2, *}$ and Iraide Mardones-Pérez ${ }^{1}$ \\ 1 Departamento de Matemáticas, Universidad del País Vasco-Euskal Herriko Unibertsitatea, Apdo. 644, \\ 48080 Bilbao, Spain; leire.legarreta@ehu.eus (L.L.); iraide.mardones@ehu.eus (I.M.-P.) \\ 2 Departamento de Matemáticas, Institute for Advanced Materials INAMAT, Universidad Pública de Navarra, \\ Campus de Arrosadía, 31006 Pamplona, Spain \\ * Correspondence: ilizasoain@unavarra.es
}

Received: 23 August 2017; Accepted: 17 September 2017; Published: 20 September 2017

\begin{abstract}
Aggregation functions are mathematical operators that merge given data in order to obtain a global value that preserves the information given by the data as much as possible. In most practical applications, this value is expected to be between the infimum and the supremum of the given data, which is guaranteed only when the aggregation functions are idempotent. Ordered weighted averaging (OWA) operators are particular cases of this kind of function, with the particularity that the obtained global value depends on neither the source nor the expert that provides each datum, but only on the set of values. They have been classified by means of the orness-a measurement of the proximity of an OWA operator to the OR-operator. In this paper, the concept of orness is extended to the framework of idempotent aggregation functions defined both on the real unit interval and on a complete lattice with a local finiteness condition.
\end{abstract}

Keywords: aggregation functions; lattice operators; idempotence; orness

\section{Introduction}

Aggregation functions [1] are a family of operators that allow us to fuse data-either quantitative or qualitative-in order to obtain a global value that captures the information of the given data as faithfully as possible.

These functions have been widely used in decision making [2,3], where it is necessary to merge the different opinions of several experts, or in image processing tasks [4], where the values of different pixels must be fused in order to obtain a single one.

OWA (Ordered weighted averaging) operators, defined by Yager in [5] for real values, are a kind of aggregation function that include the most widely used ones, such as the arithmetic mean or the order statistics. An OWA operator is simply a weighted average of data which have been previously ordered in a descending way. The main property of OWA operators is the symmetry, which makes them independent of the order in which the data are provided.

Real OWA operators have been classified by Yager in [6] by means of the orness-a measurement of their proximity to the OR-operator. In this way, the orness of the OR-operator is 1 , while the orness of the AND-operator is 0 and the orness of the arithmetic mean is equal to $1 / 2$.

In [7], OWA operators have been extended from $[0,1]$ to a complete lattice $\left(L, \leq_{L}\right)$ endowed with both a t-norm $T$ and a t-conorm $S$. The concept of orness has also been generalized to this kind of operator in two ways. On the one hand, a qualitative orness has been defined for OWA operators when the lattice $L$ is finite [4]. On the other hand, when the lattice satisfies a weaker locally-finiteness condition, each OWA operator may be assigned a quantitative orness [8].

The present paper analyzes the possibility of extending both concepts of orness to the wider set of all the aggregation functions lying between the AND and the OR-operators, for which the measurement of their proximity to the OR-operator has sense. These aggregation functions-not necessarily 
symmetric - are precisely those that are idempotent (see Remark 1). Such maps will be the object of our study when they are defined both on the real case and on some lattices that satisfy the necessary conditions of finiteness.

The extension of the concept of orness to all of the idempotent aggregation functions makes it possible to classify two wide classes of operators: the class consisting of all the Choquet integrals defined on the real unit interval on the one hand, and all the discrete Sugeno integrals defined on an appropriate lattice on the other hand.

The rest of the paper is organized as follows. Section 2 presents the preliminary concepts that will be used further. In Section 3, Yager's concept of orness is extended to idempotent aggregation functions defined on the real unit interval. Section 4 generalizes the concept of qualitative orness to idempotent aggregation functions defined on finite lattices. The extension of the concept of quantitative orness to this kind of function is analyzed in Section 5 where they are defined on lattices with a local finiteness condition. The paper finishes with some conclusions and references.

\section{Preliminaries}

In this paper, $\left(L, \leq_{L}\right)$ denotes a complete lattice (i.e., a partially ordered set in which all subsets have both a supremum and an infimum). The least element of $L$ is denoted by $0_{L}$ and the greatest one by $1_{L}$. When $L$ is finite, we will refer to $\left(L, \leq_{L}\right)$ as a finite bounded lattice. In some cases, we will require the lattice $\left(L, \leq_{L}\right)$ to be distributive, which means that any of the following two equivalent properties holds:

$$
\begin{aligned}
& a \wedge(b \vee c)=(a \wedge b) \vee(a \wedge c) \text { for any } a, b, c \in L \\
& a \vee(b \wedge c)=(a \vee b) \wedge(a \vee c) \text { for any } a, b, c \in L
\end{aligned}
$$

A map $T: L \times L \rightarrow L$ (resp. $S: L \times L \rightarrow L$ ) is said to be a $t$-norm (resp. $t$-conorm) on $\left(L, \leq_{L}\right)$ if it is commutative, associative, increasing in each component, and has a neutral element $1_{L}\left(\right.$ resp. $\left.0_{L}\right)$. For any $n>2, S\left(a_{1}, \ldots, a_{n}\right)$ will be used to denote $S\left(S\left(\ldots\left(S\left(S\left(a_{1}, a_{2}\right), a_{3}\right), \ldots\right), a_{n-1}\right), a_{n}\right)$.

Throughout this paper, $\left(L, \leq_{L}, T, S\right)$ will denote a complete lattice endowed with a t-norm and a t-conorm.

Recall that an $n$-ary aggregation function is a map $M: L^{n} \rightarrow L$ such that:

(i) $M\left(a_{1}, \ldots, a_{n}\right) \leq_{L} M\left(a_{1}^{\prime}, \ldots, a_{n}^{\prime}\right)$ whenever $a_{i} \leq_{L} a_{i}^{\prime}$ for $1 \leq i \leq n$.

(ii) $\quad M\left(0_{L}, \ldots, 0_{L}\right)=0_{L}$ and $M\left(1_{L}, \ldots, 1_{L}\right)=1_{L}$.

The function $M$ is said to be idempotent if $M(a, \ldots, a)=a$ for every $a \in L$. Besides, $M$ is said to be symmetric if for every permutation $\sigma$ of the set $\{1, \ldots, n\}$, the equality $M\left(a_{1}, \ldots, a_{n}\right)=$ $M\left(a_{\sigma(1)}, \ldots, a_{\sigma(n)}\right)$ holds for every $\left(a_{1}, \ldots, a_{n}\right) \in L^{n}$.

For example, the AND-operator, the OR-operator and, for $L=[0,1]($ or $L \subset \mathbb{R}$ ) the arithmetic mean are symmetric idempotent aggregation functions.

Remark 1. Note that by using property (i) of the definition of aggregation function, we have the following equivalence:

$$
\begin{aligned}
M \text { is idempotent } \Longleftrightarrow \quad & \inf \left\{a_{1}, \ldots, a_{n}\right\} \leq_{L} M\left(a_{1}, \ldots, a_{n}\right) \leq_{L} \sup \left\{a_{1}, \ldots, a_{n}\right\}, \\
& \text { for any }\left(a_{1}, \ldots, a_{n}\right) \in L^{n},
\end{aligned}
$$

i.e., the aggregation function $M$ is idempotent iff it lies in between the AND and the OR-operators. Indeed, if $M$ is idempotent, $\inf \left\{a_{1}, \ldots, a_{n}\right\}=M\left(\inf \left\{a_{1}, \ldots, a_{n}\right\}, \ldots, \inf \left\{a_{1}, \ldots, a_{n}\right\}\right) \leq M\left(a_{1}, \ldots, a_{n}\right) \leq$ $M\left(\sup \left\{a_{1}, \ldots, a_{n}\right\}, \ldots, \sup \left\{a_{1}, \ldots, a_{n}\right\}\right)=\sup \left\{a_{1}, \ldots, a_{n}\right\}$. Conversely, for any a $\in L$, $a=\inf \{a, \ldots, a\} \leq M(a, \ldots, a) \leq \sup \{a, \ldots, a\}=a$. 
A particular case of idempotent symmetric aggregation functions are OWA operators, which were defined by Yager for values in the real unit interval $[0,1]$ as follows:

Definition 1 (Yager [5]). For each weighting vector $\alpha=\left(\alpha_{1}, \ldots, \alpha_{n}\right) \in[0,1]^{n}$, with $\alpha_{1}+\cdots+\alpha_{n}=1$, the map $F_{\alpha}:[0,1]^{n} \rightarrow[0,1]$ given by

$$
F_{\alpha}\left(a_{1}, \ldots, a_{n}\right)=\alpha_{1} a_{\sigma(1)}+\cdots+\alpha_{n} a_{\sigma(n)}
$$

is called an ordered weighted averaging operator or OWA operator, where the $n$-tuple $\left(a_{\sigma(1)}, \ldots, a_{\sigma(n)}\right)$ is a rearrangement of the set $\left(a_{1}, \ldots, a_{n}\right)$ of inputs such that $a_{\sigma(1)} \geq \cdots \geq a_{\sigma(n)}$.

Note that both the AND-operator and the OR-operator are some examples of OWA operators, provided respectively by the weighting vector $(0, \ldots, 0,1)$ and by the weighting vector $(1,0, \ldots, 0)$. The arithmetic mean is also an OWA operator, provided by the weighting vector $(1 / n, \ldots, 1 / n)$.

OWA operators were generalized to complete lattices in [7] in the following way.

Definition 2. Let $\left(L, \leq_{L}, T, S\right)$ be a complete lattice endowed with a t-norm and a t-conorm. A vector $\alpha=\left(\alpha_{1}, \ldots, \alpha_{n}\right) \in L^{n}$ is said to be $a$ distributive weighting vector if conditions $S\left(\alpha_{1}, \ldots, \alpha_{n}\right)=1_{L}$ and

$$
T\left(a, S\left(\alpha_{1}, \ldots, \alpha_{n}\right)\right)=S\left(T\left(a, \alpha_{1}\right), \ldots, T\left(a, \alpha_{n}\right)\right) \text { for any } a \in L
$$

are satisfied.

Definition 3 ([7]). Let $\left(L, \leq_{L}, T, S\right)$ be a complete lattice endowed with a $t$-norm and a t-conorm. For each distributive weighting vector $\alpha=\left(\alpha_{1}, \ldots, \alpha_{n}\right) \in L^{n}$, the $n$-ary OWA operator $F_{\alpha}: L^{n} \rightarrow L$ is defined by

$$
F_{\alpha}\left(a_{1}, \ldots, a_{n}\right)=S\left(T\left(\alpha_{1}, b_{1}\right), \ldots, T\left(\alpha_{n}, b_{n}\right)\right),
$$

where for each $\left(a_{1}, \ldots, a_{n}\right) \in L^{n}$, the elements $b_{n} \leq_{L} \cdots \leq_{L} b_{1}$ are calculated by means of the $n$-ary $k$-th statistics $\left\{A_{k}: L^{n} \rightarrow L \mid 1 \leq k \leq n\right\}$ given below:

- $\quad b_{1}=A_{1}\left(a_{1}, \ldots, a_{n}\right)=a_{1} \vee \cdots \vee a_{n} \in L$.

- $b_{2}=A_{2}\left(a_{1}, \ldots, a_{n}\right)=\left(\left(a_{1} \wedge a_{2}\right) \vee \cdots \vee\left(a_{1} \wedge a_{n}\right)\right) \vee\left(\left(a_{2} \wedge a_{3}\right) \vee \cdots \vee\left(a_{2} \wedge a_{n}\right)\right) \vee \cdots \vee\left(a_{n-1} \wedge\right.$ $\left.a_{n}\right) \in L$

- $\quad b_{k}=A_{k}\left(a_{1}, \ldots, a_{n}\right)=\bigvee\left\{a_{j_{1}} \wedge \cdots \wedge a_{j_{k}} \mid j_{1}<\cdots<j_{k} \in\{1, \ldots, n\}\right\} \in L$

- $\quad b_{n}=A_{n}\left(a_{1}, \ldots, a_{n}\right)=a_{1} \wedge \cdots \wedge a_{n} \in L$.

It is worth mentioning that when $\left(L, \leq_{L}\right)$ is a distributive lattice and the t-norm and t-conorm considered on $L$ are, respectively, the meet and the join (i.e., $T=\wedge$ and $S=V$ ), OWA operators are particular cases of a wider class of idempotent aggregation functions: the discrete Sugeno integrals.

Definition 4 ([9]). Let $\left(L, \leq_{L}\right)$ be a complete lattice, $P_{n}=\{1, \ldots, n\}$ and let $\mathcal{P}_{n}$ be the set of all the subsets of $P_{n}$.

(i) An L-fuzzy measure on $P_{n}$ is a map $\mu: \mathcal{P}_{n} \rightarrow L$ with $\mu(\varnothing)=0_{L}, \mu\left(P_{n}\right)=1_{L}$ and $\mu(E) \leq \mu(F)$ whenever $E \subseteq F \subseteq P_{n}$.

In particular, if $L=[0,1]$, it is called a fuzzy measure [10,11]. 
(ii) The discrete Sugeno integral $S_{\mu}: L^{n} \rightarrow L$ is given by

$$
S_{\mu}\left(a_{1}, \ldots, a_{n}\right)=\bigvee\left\{\bigwedge\left\{a_{i} \mid i \in X\right\} \wedge \mu(X): X \in \mathcal{P}_{n}\right\}
$$

In fact, when $L$ is a distributive complete lattice, a discrete Sugeno integral is an OWA operator if and only if it is symmetric (see [7]).

\section{Orness for Idempotent Aggregation Functions Defined on the Real Unit Interval}

The great deal of weighting vectors that can define an OWA operator on the real interval $[0,1]$ makes difficult the election for each particular situation.

With the purpose of classifying OWA operators defined on the real unit interval, in [6] Yager introduced an orness measure for each OWA operator $F_{\alpha}$. This measure depends only on the weighting vector $\alpha=\left(\alpha_{1}, \ldots, \alpha_{n}\right)$, in the following way:

$$
\operatorname{orness}\left(F_{\alpha}\right)=\frac{1}{n-1} \sum_{i=1}^{n}(n-i) \alpha_{i}
$$

It is easy to check that the orness of each OWA operator is a real value situated between 0 , corresponding to the AND-operator given by the infimum, and 1, corresponding to the OR-operator given by the supremum. In general, the orness is a measure of the proximity of each OWA operator to the OR-operator. For instance, the orness of the arithmetic mean, provided by the weighting vector $(1 / n, \ldots, 1 / n)$, is equal to $1 / 2$.

In order to extend this concept to any idempotent aggregation function, note that for any OWA operator $F_{\alpha}:[0,1]^{n} \rightarrow[0,1]$, we can write

$$
\operatorname{orness}\left(F_{\alpha}\right)=F_{\alpha}\left(1, \frac{n-2}{n-1}, \cdots, \frac{1}{n-1}, 0\right),
$$

which leads to the following generalization.

Definition 5. Let $F:[0,1]^{n} \rightarrow[0,1]$ be an idempotent aggregation function. Define

$$
\operatorname{orness}(F)=F\left(1, \frac{n-2}{n-1}, \cdots, \frac{1}{n-1}, 0\right) \text {. }
$$

Remark 2. (i) Since F always takes values in $[0,1]$, it is clear that $0 \leq$ orness $(F) \leq 1$. Moreover, the orness of the AND-operator is equal to 0 , whereas the orness of the OR-operator is 1 .

(ii) It is immediate to see that orness $(F) \leq \operatorname{orness}(G)$ if $F \leq G$ (with the pointwise order). However, as Example 1 below will show, the converse is not true.

(iii) Definition 5 could have been given for any aggregation function $F:[0,1]^{n} \rightarrow[0,1]$, but it does not make any sense to measure the proximity of $F$ to the OR-operator when there does not exist any order relation between them.

Moreover, if $F\left(a_{1}, \ldots, a_{n}\right) \leq \inf \left(a_{1}, \ldots, a_{n}\right)$ for all $\left(a_{1}, \ldots, a_{n}\right)$, we would have orness $(F)=0$. Similarly, if $F\left(a_{1}, \ldots, a_{n}\right) \geq \sup \left(a_{1}, \ldots, a_{n}\right)$ for all $\left(a_{1}, \ldots, a_{n}\right)$, then orness $(F)$ would be equal to 1 .

For the aforementioned reasons, we have extended the definition of orness only to the class of idempotent aggregation functions, which are exactly those lying between the AND and the OR-operators.

The previous extension of the concept of orness makes it possible to classify a wider kind of idempotent aggregation functions which include OWA operators: the so called discrete Choquet integrals. Their definition is as follows: 
Definition 6 ([12]). Let $m: \mathcal{P}_{n} \rightarrow[0,1]$ be a fuzzy measure. The discrete Choquet integral with respect to $m$ is the map $C_{m}:[0,1]^{n} \rightarrow[0,1]$ defined by

$$
C_{m}\left(x_{1}, \ldots, x_{n}\right)=\sum_{i=1}^{n} x_{\sigma(i)}(m(\{\sigma(i), \ldots, \sigma(n)\})-m(\{\sigma(i+1), \ldots, \sigma(n)\}))
$$

where $\sigma:\{1, \ldots, n\} \rightarrow\{1, \ldots, n\}$ is a permutation such that $x_{\sigma(1)} \leq \cdots \leq x_{\sigma(n)}$ and $\{\sigma(n+1), \sigma(n)\}=\varnothing$ by convention.

Remark 3. As was shown in [13], a discrete Choquet integral is an OWA operator if and only if it is symmetric. The latter is equivalent to the fuzzy measure being symmetric, i.e., $m(E)=m(F)$ for any $E, F \subseteq P_{n}$ such that $\operatorname{card}(E)=\operatorname{card}(F)$.

Example 1. Let $n=4$ and consider the fuzzy measures $m, v: \mathcal{P}_{4} \rightarrow[0,1]$ given by

$$
\begin{aligned}
& m(\{1\})=m(\{4\})=0=v(\{1\})=v(\{2\}) \\
& m(\{2\})=m(\{3\})=\frac{1}{4}=v(\{3\})=v(\{4\}) \\
& m(\{i, j\})=\frac{1}{3}=v(\{i, j\}) \quad 1 \leq i<j \leq 4 ; \\
& m(\{i, j, k\})=\frac{1}{2}=v(\{i, j, k\}) \quad 1 \leq i<j<k \leq 4 ; \\
& m\left(P_{4}\right)=1=v\left(P_{4}\right) .
\end{aligned}
$$

The orness of each of the corresponding non-symmetric discrete Choquet integrals $C_{m}, C_{v}:[0,1]^{4} \rightarrow[0,1]$ is given by

$$
\begin{aligned}
\operatorname{orness}\left(C_{m}\right) & =C_{m}\left(1, \frac{2}{3}, \frac{1}{3}, 0\right) \\
& =\frac{1}{3}\left(\frac{1}{2}-\frac{1}{3}\right)+\frac{2}{3}\left(\frac{1}{3}-0\right)=\frac{5}{18} \\
& =C_{v}\left(1, \frac{2}{3}, \frac{1}{3}, 0\right) \\
& =\operatorname{orness}\left(C_{v}\right)
\end{aligned}
$$

so, in particular, orness $\left(C_{v}\right) \leq$ orness $\left(C_{m}\right)$. However,

$$
\begin{aligned}
& C_{m}\left(0,0, \frac{1}{2}, 1\right)=\frac{1}{2}\left(\frac{1}{3}-0\right)=\frac{1}{6} \\
& C_{v}\left(0,0, \frac{1}{2}, 1\right)=\frac{1}{2}\left(\frac{1}{3}-\frac{1}{4}\right)+1\left(\frac{1}{4}-0\right)=\frac{7}{24}
\end{aligned}
$$

and therefore, $C_{v} \leq C_{m}$. The latter shows that the converse of Remark 2(ii) is not true.

\section{Qualitative Orness for Idempotent Aggregation Functions Defined on Finite Lattices}

In this section, $\left(L, \leq_{L}\right)$ will be a bounded finite lattice.

In order to classify OWA operators when they are defined on this kind of lattice, a qualitative orness was introduced in [4] generalizing the one given by Yager for the real case. In this section, this concept is extended to any idempotent aggregation function defined on $\left(L, \leq_{L}\right)$. Note that, by [4], Remark 3, the definition given in [4] can be reformulated so that it still has sense in this wider context.

Definition 7. Let $L=\left\{a_{1}, \ldots, a_{l}\right\}$ be a finite bounded lattice and let $1_{L}=b_{1} \geq_{L} \cdots \geq_{L} b_{l}=0_{L}$ be the descending chain introduced in Definition 3, involving all the elements of the lattice. For any idempotent n-ary aggregation function $F: L^{n} \rightarrow L$, a qualitative orness measure is calculated by means of a descending chain $d_{1} \geq_{L} \cdots \geq_{L} d_{n}$ that consists of some equidistant elements in the lattice, which are performed following some steps:

(i) Call $s=l(n-1)$. 
(ii) Consider the descending chain $c_{1} \geq_{L} \cdots \geq_{L} c_{S}$ defined by $c_{1}=\cdots=c_{n-1}=b_{1} ; c_{n}=\cdots=c_{2(n-1)}=b_{2} ; \ldots ; c_{(l-1)(n-1)+1}=\cdots=c_{l(n-1)}=b_{l}$.

Note that $c_{1}=b_{1}=1_{L}$ and $c_{l(n-1)}=b_{l}=0_{L}$.

(iii) Build a descending subchain of $\left\{c_{1}, \ldots, c_{s}\right\}, d_{1} \geq_{L} \cdots \geq_{L} d_{n}$ by means of

$$
d_{1}=1_{L}, d_{2}=c_{l}, d_{3}=c_{2 l}, \ldots, d_{n}=c_{(n-1) l}=0_{L}
$$

i.e., $d_{1}=1_{L}$, and for each $j \in\{1, \ldots, n-1\}, d_{j+1}=c_{j l}=b_{k}$ with $k=1+\left\lfloor\frac{j l-1}{n-1}\right\rfloor$, where the symbol $\left\lfloor\frac{a}{b}\right\rfloor$ denotes the integer part of $\frac{a}{b}$.

(iv) Call orness $(F)=F\left(d_{1}, \ldots, d_{n}\right)$.

As in [4], we have the following observations.

Remark 4. (i) Note that the same chain $d_{1} \geq_{L} \cdots \geq_{L} d_{n}$ is obtained if we let $s$ be any common multiple of $\{l, n-1\}$. In that case, if $s=$ le with $e \in \mathbb{N}$, the chain $c_{1} \geq_{L} \cdots \geq_{L} c_{S}$ must be

$c_{1}=\cdots=c_{e}=b_{1} ; c_{e+1}=\cdots=c_{2 e}=b_{2} ; \ldots ; c_{(l-1) e+1}=\cdots=c_{l e}=b_{l}$ and, if $s=(n-1) h$ with $h \in \mathbb{N}$, then the chain $d_{1} \geq_{L} \cdots \geq_{L} d_{n}$ must be

$$
d_{1}=1_{L}, d_{2}=c_{h}, d_{3}=c_{2 h}, \ldots, d_{n}=c_{(n-1) h}=0_{L}
$$

Therefore, the chain $d_{1} \geq_{L} \cdots \geq_{L} d_{n}$ obtained in this way is the same as that obtained in Definition 7. Indeed, for each $j \in\{1, \ldots, n-1\}, d_{j+1}=c_{j h}=b_{k}$ with $k=1+\left\lfloor\frac{j h-1}{e}\right\rfloor$.

(ii) When the aggregation function is an OWA operator, the previous definition agrees with that given in [4] for OWA operators. Hence, in particular, the orness corresponding to the AND-operator is 0, and the one corresponding to the OR-operator is 1 .

(iii) Definition 7 could have been given for any aggregation function (not necessarily idempotent), but the same reasons explained in Remark 2(iii) lead us to consider this restriction.

Example 2. Let $L=\left\{0_{L}, a, b, c, 1_{L}\right\}$ be a partially ordered set with the relation given by $0_{L} \leq a \leq b \leq 1_{L}$ and $0_{L} \leq c \leq 1_{L}$. Note that $\left(L, \leq_{L}\right)$ is a non-distributive lattice with $l=5$. Consider the discrete Sugeno integral $S_{\mu}: L^{3} \rightarrow$ L defined by means of the L-fuzzy measure $\mu: \mathcal{P}_{3} \rightarrow L$ with

$$
\begin{gathered}
\mu(\{1\})=\mu(\{3\})=0_{L} ; \mu(\{2\})=a ; \\
\mu(\{i, j\})=a, 1 \leq i<j \leq 3 ; \mu(\{1,2,3\})=1_{L}, \\
S_{\mu}\left(a_{1}, a_{2}, a_{3}\right)=\left(a_{2} \wedge a\right) \vee\left(a_{1} \wedge a_{2} \wedge a\right) \vee\left(a_{1} \wedge a_{3} \wedge a\right) \vee\left(a_{2} \wedge a_{3} \wedge a\right) \vee\left(a_{1} \wedge a_{2} \wedge a_{3}\right) .
\end{gathered}
$$

Note that since $S_{\mu}$ is not symmetric, it is not an OWA operator. Nevertheless, we can compute its qualitative orness with the new definition we have just given. In order to calculate it, we follow the next steps:

Find $b_{1}=1_{L}, b_{2}=1_{L}, b_{3}=a, b_{4}=0_{L}, b_{5}=0_{L}$.

Call $s=l(n-1)=5 \cdot 2=10$.

Consider the descending chain $c_{1} \geq_{L} \cdots \geq_{L} c_{10}$ defined by

$c_{1}=c_{2}=b_{1} ; c_{3}=c_{4}=b_{2} ; c_{5}=c_{6}=b_{3} ; c_{7}=c_{8}=b_{4} ; c_{9}=c_{10}=b_{5}$.

Build the descending subchain $d_{1} \geq_{L} d_{2} \geq_{L} d_{3}$, by means of

$d_{1}=1_{L} ; d_{2}=c_{5}=a ; d_{3}=c_{10}=0_{L}$. 
Therefore, orness $\left(S_{\mu}\right)=S_{\mu}\left(1_{L}, a, 0_{L}\right)=a$.

In [14] it is shown that the class of all the $n$-ary aggregation functions defined on a complete lattice $\left(L, \leq_{L}\right)$-denoted by $\mathcal{A}_{n}(L)$-is also a complete lattice with the pointwise order relation; i.e., with the relation $\leq$ defined for any $F, G \in \mathcal{A}_{n}(L)$ as $F \leq G$ if and only if $F\left(a_{1}, \ldots, a_{n}\right) \leq G\left(a_{1}, \ldots, a_{n}\right)$ for every $\left(a_{1}, \ldots, a_{n}\right) \in L^{n}$.

The smallest and the greatest aggregation functions in $\mathcal{A}_{n}(L)$ are, respectively (see [15]):

$$
\begin{aligned}
& A_{\perp}\left(a_{1}, \ldots, a_{n}\right)=\left\{\begin{array}{lr}
1, & \text { if } a_{i}=1 \text { for all } 1 \leq i \leq n \\
0, & \text { otherwise }
\end{array}\right. \\
& A_{\top}\left(a_{1}, \ldots, a_{n}\right)=\left\{\begin{array}{lr}
0, & \text { if } a_{i}=0 \text { for all } 1 \leq i \leq n \\
1, & \text { otherwise }
\end{array}\right.
\end{aligned}
$$

We use the symbols $\wedge, \vee$ to denote both the meet and the join on $L$ and the following operations on $\mathcal{A}_{n}(L)$ :

$$
\begin{aligned}
& (F \wedge G)\left(a_{1}, \ldots, a_{n}\right)=F\left(a_{1}, \ldots, a_{n}\right) \wedge G\left(a_{1}, \ldots, a_{n}\right) \\
& (F \vee G)\left(a_{1}, \ldots, a_{n}\right)=F\left(a_{1}, \ldots, a_{n}\right) \vee G\left(a_{1}, \ldots, a_{n}\right),
\end{aligned}
$$

for any $\left(a_{1}, \ldots, a_{n}\right) \in L^{n}$.

Note [14] that if $\left(L, \leq_{L}\right)$ is a distributive lattice, then $\left(\mathcal{A}_{n}(L), \leq\right)$ is a distributive lattice as well.

Proposition 1. Let $\left(L, \leq_{L}\right)$ be a bounded finite lattice and consider $F, G: L^{n} \rightarrow L$, idempotent aggregation functions.

(i) If $F \leq G$, then orness $(F) \leq$ orness $(G)$.

(ii) Both $F \wedge G$ and $F \vee G$ are idempotent.

(iii) It holds that

$$
\begin{aligned}
& \operatorname{orness}(F \wedge G)=\operatorname{orness}(F) \wedge \operatorname{orness}(G) \\
& \operatorname{orness}(F \vee G)=\operatorname{orness}(F) \vee \operatorname{orness}(G)
\end{aligned}
$$

Proof. Consider the chain $d_{1} \geq_{L} \cdots \geq_{L} d_{n}$ obtained in Definition 7.

(i) Since $F \leq G$, then orness $(F)=F\left(d_{1}, \ldots, d_{n}\right) \leq_{L} G\left(d_{1}, \ldots, d_{n}\right)=\operatorname{orness}(G)$.

(ii) For any $a \in L,(F \wedge G)(a, \ldots, a)=F(a, \ldots, a) \wedge G(a, \ldots, a)=a \wedge a=a$ by using the idempotency of $F$ and $G$. In the same way, the idempotency of $F \vee G$ can be proved.

(iii) By Definition 7, orness $(F \wedge G)=(F \wedge G)\left(d_{1}, \ldots, d_{n}\right)=F\left(d_{1}, \ldots, d_{n}\right) \wedge G\left(d_{1}, \ldots, d_{n}\right)=$ $\operatorname{orness}(F) \wedge$ orness $(G)$. Analogously, orness $(F \vee G)=(F \vee G)\left(d_{1}, \ldots, d_{n}\right)=F\left(d_{1}, \ldots, d_{n}\right) \vee$ $G\left(d_{1}, \ldots, d_{n}\right)=\operatorname{orness}(F) \vee$ orness $(G)$.

Remark 5. As for the orness messure of Section 3, the converse of Proposition 1 (i) is not true. Indeed, let $L=\left\{0_{L}, a, b, c, 1_{L}\right\}$ and $S_{\mu}$ be, respectively, the lattice and the discrete Sugeno integral defined in Example 2.

Consider now the non-symmetric discrete Sugeno integral $S_{v}: L^{3} \rightarrow L$ defined by means of the L-fuzzy measure $v: \mathcal{P}_{3} \rightarrow$ L with

$$
\begin{gathered}
v(\{i\})=0_{L}, 1 \leq i \leq 3 ; v(\{1,2\})=a, v(\{1,3\})=v(\{2,3\})=c ; v(\{1,2,3\})=1_{L}, \\
S_{v}\left(a_{1}, a_{2}, a_{3}\right)=\left(a_{1} \wedge a_{2} \wedge a\right) \vee\left(a_{2} \wedge a_{3} \wedge c\right) \vee\left(a_{1} \wedge a_{3} \wedge c\right) \vee\left(a_{1} \wedge a_{2} \wedge a_{3}\right) .
\end{gathered}
$$


Therefore, $\operatorname{orness}\left(S_{v}\right)=S_{\nu}\left(1_{L}, a, 0_{L}\right)=a=\operatorname{orness}\left(S_{\mu}\right)$.

However, $S_{\mu}(c, a, c)=a \not \leq c=S_{v}(c, a, c)$.

\section{Quantitative Orness for Idempotent Aggregation Functions}

In this section, $\left(L, \leq_{L}\right)$ will be a complete lattice satisfying the following additional condition of local finiteness, which is called maximal finite chain condition or simply condition (MFC):

(MFC) For any $a, b \in L$ with $a \leq_{L} b$, there exists some maximal chain with a finite length, named $l$, between $a$ and $b$,

$$
a=a_{0}<_{L} a_{1}<_{L} \cdots<_{L} a_{l}=b
$$

The maximality means that for any $0 \leq i \leq l-1$ there is no $c \in L$ with $a_{i}<_{L} c<_{L} a_{i+1}$. Obviously, if $a=b$, the maximal chain has length $l=0$. The length of the shortest maximal chain between $a$ and $b$-which will be denoted by $d(a, b)$ — will be called distance from $a$ to $b$.

Remark 6. Note that any finite lattice satisfies condition (MFC).

A quantitative orness was defined in [8] in the following way for any OWA operator defined on $\left(L, \leq_{L}, T, S\right)$.

Definition 8 ([8]). Let $\left(L, \leq_{L}, T, S\right)$ be a complete lattice, endowed with a t-norm and a t-conorm satisfying condition (MFC). For any distributive weighting vector, $\alpha=\left(\alpha_{1}, \ldots, \alpha_{n}\right) \in L^{n}$, define the qualitative quantifier $Q_{\alpha}:\{0,1, \ldots, n\} \rightarrow$ L by means of

$$
\begin{aligned}
& Q_{\alpha}(0)=0_{L} \\
& Q_{\alpha}(k)=S\left(\alpha_{1}, \ldots, \alpha_{k}\right) \text { for } k=1, \ldots, n .
\end{aligned}
$$

Definition 9 ([8]). Let $\left(L, \leq_{L}, T, S\right)$ be a complete lattice satisfying condition (MFC). For any distributive weighting vector $\alpha=\left(\alpha_{1}, \ldots, \alpha_{n}\right) \in L^{n}$, let $F_{\alpha}$ be the $n$-ary OWA operator associated to it. Consider the qualitative quantifier $Q_{\alpha}:\{0,1, \ldots, n\} \rightarrow L$ defined in Definition 8. For each $k=1, \ldots, n$, call $m(k)=d\left(Q_{\alpha}(k-1), Q_{\alpha}(k)\right)$. If $m=m(1)+\cdots+m(n)$, then define

$$
\operatorname{orness}\left(F_{\alpha}\right)=\frac{1}{n-1} \sum_{i=1}^{n}(n-i) \frac{m(i)}{m} \text {. }
$$

The following remark contains the key points that allow us to generalize this concept to the setting of idempotent aggregation functions.

Remark 7 ([8]). Let $\alpha=\left(\alpha_{1}, \ldots, \alpha_{n}\right) \in L^{n}$ be a distributive weighting vector in $\left(L, \leq_{L}, T, S\right)$. Then

(i) $Q_{\alpha}$ is a monotonically increasing function.

(ii) For any $k=1, \ldots, n, Q_{\alpha}(k)=F_{\alpha}\left(1_{L}, \ldots, 1_{L}, 0_{L}, \ldots, 0_{L}\right)$.

(iii) Consequently, if $\beta=\left(\beta_{1}, \ldots, \beta_{n}\right) \in L^{n}$ is a distributive weighting vector in $\left(L, \leq_{L}\right)$ with $F_{\alpha}=F_{\beta}$, then $Q_{\alpha}=Q_{\beta}$.

Definition 10. Let $\left(L, \leq_{L}\right)$ be a complete lattice satisfying condition (MFC). For any idempotent aggregation function $F: L^{n} \rightarrow L$, consider the qualitative quantifier $Q:\{0,1, \ldots, n\} \rightarrow L$ defined by means of

$$
\begin{aligned}
& Q(0)=0_{L} \\
& Q(k)=F\left(1_{L}, \ldots, 1_{L}, 0_{L}, \ldots, 0_{L}\right) \text { for } k=1, \ldots, n .
\end{aligned}
$$


For each $k=1, \ldots, n$, call $m(k)=d(Q(k-1), Q(k))$. If $m=m(1)+\cdots+m(n)$, then define

$$
\operatorname{orness}(F)=\frac{1}{n-1} \sum_{i=1}^{n}(n-i) \frac{m(i)}{m} .
$$

Remark 8. (i) Note that for arbitrary idempotent aggregation functions, the definition of orness does not directly depend on the t-norm and the $t$-conorm considered on the lattice $L$.

(ii) When the aggregation function is an OWA operator, Remark 7(ii) shows that the previous definition agrees with the one given in [8] for OWA operators. In particular, as shown in [8], Proposition 3.6, the orness corresponding to the AND-operator is 0 and the one corresponding to the OR-operator is 1.

(iii) Observe that, contrary to the case of OWA operators (see [8]), Proposition 3.6(ii), there might be idempotent aggregation functions different from the AND-operator whose orness is 0 . For instance, take $L=\left\{0_{L}, c, 1_{L}\right\}$ with $0_{L} \leq c \leq 1_{L}$. Define $F: L^{3} \rightarrow L$ as $F\left(a_{1}, a_{2}, a_{3}\right)=a_{3}$. Then, $F$ is an idempotent aggregation function different from the AND-operator whose orness is 0 .

(iv) Definition 10 could have been given for any aggregation function, not necessarily idempotent, but the same reasons explained in Remark 2(iii) lead us to consider this restriction.

Proposition 2. Let $\left(L, \leq_{L}\right)$ be a complete lattice satisfying condition (MFC). For any idempotent aggregation function $F: L^{n} \rightarrow L$, it is satisfied that orness $(F) \in[0,1]$.

Proof. Clearly, orness $(F) \geq 0$. Moreover,

$$
\operatorname{orness}(F)=\frac{1}{m(n-1)} \sum_{i=1}^{n}(n-i) m(i) \leq \frac{1}{m(n-1)} \sum_{i=1}^{n}(n-1) m(i)=1
$$

as desired.

Example 3. Let $L=\left\{0_{L}, a, b, 1_{L}\right\} \cup\left\{c_{i}: i \in \mathbb{N}\right\}$, with $c_{i} \neq c_{j}$ for $i \neq j$, be the lattice described as follows: $0_{L} \leq a \leq b \leq 1_{L}$ and $0_{L} \leq c_{i} \leq 1_{L}$ for any $i \in \mathbb{N}$. Then, $L$ is a non-finite lattice satisfying condition (MFC). Consider the discrete Sugeno integral $S_{\mu}: L^{3} \rightarrow$ L defined by means of the L-fuzzy measure $\mu: \mathcal{P}_{3} \rightarrow L$ with

$$
\begin{gathered}
\mu(\{1\})=\mu(\{3\})=0_{L} ; \mu(\{2\})=a ; \\
\mu(\{i, j\})=a, 1 \leq i<j \leq 3 ; \mu(\{1,2,3\})=1_{L}, \\
S_{\mu}\left(a_{1}, a_{2}, a_{3}\right)=\left(a_{2} \wedge a\right) \vee\left(a_{1} \wedge a_{2} \wedge a\right) \vee\left(a_{1} \wedge a_{3} \wedge a\right) \vee\left(a_{2} \wedge a_{3} \wedge a\right) \vee\left(a_{1} \wedge a_{2} \wedge a_{3}\right) .
\end{gathered}
$$

Even if $S_{\mu}$ is not an OWA operator (since it is not symmetric), we can compute its quantitative orness with the new definition we have just given. In order to calculate it, we follow these steps:

$$
\begin{aligned}
& \text { Define } Q:\{0,1,2,3\} \rightarrow L \text { by means of } Q(0)=0_{L}, Q(1)=S_{\mu}\left(1_{L}, 0_{L}, 0_{L}\right)=0_{L}, \\
& Q(2)=S_{\mu}\left(1_{L}, 1_{L}, 0_{L}\right)=a, Q(3)=S_{\mu}\left(1_{L}, 1_{L}, 1_{L}\right)=1_{L} . \\
& \text { Calculate } m(1)=d(Q(0), Q(1))=0 ; m(2)=d(Q(1), Q(2))=1 ; \\
& m(3)=d(Q(2), Q(3))=2 \text { and } m=m(1)+m(2)+m(3)=3 . \\
& \text { Therefore, orness }\left(S_{\mu}\right)=\frac{1}{2}\left(2 \cdot \frac{m(1)}{3}+1 \cdot \frac{m(2)}{3}\right)=\frac{1}{6} .
\end{aligned}
$$

\section{Conclusions}

The concept of orness defined by Yager for OWA operators on the real interval $[0,1]$ can be extended to the more general setting of idempotent aggregation functions, all of them lying between the AND and the OR-operators. This includes the class of all the discrete Choquet integrals, which are not necessarily symmetric. In addition, on finite bounded lattices, the concept of qualitative orness 
defined for OWA operators can be extended to this wide set of all the idempotent aggregation functions. Finally, for complete lattices satisfying a weaker condition of local finiteness, the concept of quantitative orness defined for OWA operators can be extended to all the idempotent aggregation functions. The latter includes the set of all the discrete Sugeno integrals defined on that kind of lattice, which are not necessarily symmetric.

For future research, the application of these concepts of orness to practical situations may be analyzed.

Acknowledgments: This work has been partially supported by the research projects MTM2015-63608-P of the Spanish Government and IT974-16 of the Basque Government.

Author Contributions: Leire Legarreta, Inmaculada Lizasoain and Iraide Mardones-Pérez contributed equally to this work.

Conflicts of Interest: The authors declare no conflict of interest.

\section{References}

1. Beliakov, G.; Pradera, A.; Calvo, T. Aggregation Functions: A Guide for Practitioners; Springer: New York, USA, 2008.

2. De Miguel, L.; Bustince, H.; Pekala, B.; Bentkowska, U.; Da Silva, I.; Bedregal, B.; Mesiar, R.; Ochoa, G. Interval-Valued Atanassov Intuitionistic OWA Aggregations Using Admissible Linear Orders and Their Application to Decision Making. IEEE Trans. Fuzzy Syst. 2016, 24, 1586-1597, doi:10.1109/TFUZZ.2016.2543744.

3. De Miguel, L.; Sesma-Sara, M.; Elkano, M.; Asiain, M.J.; Bustince, H. An algorithm for group decision making using n-dimensional fuzzy sets, admissible orders and OWA operators. Inf. Fusion 2017, 37, 126-131, doi:10.1016/j.inffus.2017.01.007.

4. Ochoa, G.; Lizasoain, I.; Paternain, D.; Bustince, H.; Pal, N.R. From Quantitative to Qualitative orness for lattice OWA operators. Int. J. Gen. Syst. 2017, doi:10.1080/03081079.2017.1319364.

5. Yager, R.R. On ordered weighting averaging aggregation operators in multicriteria decision-making. IEEE Trans. Syst. Man Cybern. 1988, 18, 183-190.

6. Yager, R.R. Families of OWA operators. Fuzzy Sets Syst. 1993, 59, 125-148.

7. Lizasoain, I.; Moreno, C. OWA operators defined on complete lattices. Fuzzy Sets Syst. 2013, 224, 36-52.

8. Paternain, D.; Ochoa, G.; Lizasoain, I.; Bustince, H.; Mesiar, R. Quantitative orness for lattice OWA operators. Inf. Fusion 2016, 30, 27-35.

9. Couceiro, M.; Marichal, J.-L. Characterizations of discrete Sugeno integrals as polynomial functions over distributive lattices. Fuzzy Sets Syst. 2010, 161, 694-707.

10. Paternain, D.; Bustince, H.; Pagola, M.; Sussner, P.; Kolesárová, A.; Mesiar, R. Capacities and overlap indexes with an application in fuzzy rule-based classification systems. Fuzzy Sets Syst. 2016, 305, 70-94.

11. Torra, V.; Narukawa, Y.; Sugeno, M. (Eds.) Non-Additive Measures, Theory and Applications (Studies in Fuzziness and Soft Computing Series 310); Springer: New York, USA, 2014.

12. Choquet, G. Theory of capacities. Ann. l'Inst. Fourier 1954, 5, 131-292.

13. Mesiar, R.; Stupnanova, A.; Yager, R. Generalizations of OWA operators. In Proceedings of the 16th World Congress of the International Fuzzy Systems Association (IFSA) and the 9th Conference of the European Society for Fuzzy Logic and Technology (EUSFLAT), Gijón, Spain, 30 June-3 July 2015.

14. Karaçal, F.; Mesiar, R. Aggregation functions on bounded lattices. Int. J. Gen. Syst. 2017, 46, 37-51.

15. Komorníková, M.; Mesiar, R. Aggregation functions on Bounded Partially Ordered Sets and their classification. Fuzzy Sets Syst. 2011, 175, 48-56.

(c) 2017 by the authors. Licensee MDPI, Basel, Switzerland. This article is an open access article distributed under the terms and conditions of the Creative Commons Attribution (CC BY) license (http:// creativecommons.org/licenses/by/4.0/). 